\title{
Box Wing: Aerodynamic Experimental Study for Applications in MAVs
}

\author{
M. Singh ${ }^{1}$, J. J. Aloor ${ }^{1}$, A. Singh ${ }^{1}$ and $\underline{\text { S. Saha }}{ }^{*}$ \\ ${ }^{1}$ Department of Aerospace Engineering; Indian Institute of Technology Kharagpur, \\ Kharagpur-721302, WB, India, \\ IEmail:mohitsiitkgp17@gmail.com; *Email:ssaha@aero.iitkgp.ac.in (Corresponding Author)
}

\begin{abstract}
Advancements in the field of aerial robotics and micro aerial vehicles (MAVs) have increased the demand for high payload capabilities. Closed wing designs like the annular wing, the joined wing, the box wing and spiroid tip devices improve the aerodynamic performance by suppressing the wingtip vortices along with an enhanced lift coefficient. A box wing may be defined as a wing that effectively has two main planes which merge at their ends so that there are no conventional wingtips. We propose the implementation of box wings as the main lifting surface for such systems. Box wings have a potential of generating lift with considerably less induced drag and delayed stall angles than monoplane wings. We study the aerodynamic aspects of a box wing model using wind tunnel tests and numerical simulations. We conducted Computational Fluid Dynamics (CFD) simulation subjecting the model to a steady flow and later analysed the vortex core using CFD tools. Wind tunnel measurements of the forces were obtained using sting balance. Furthermore, polyester thread tufts and smoke flow visualisation were performed to understand the qualitative behaviour of the scaled model in the open to atmosphere, suction type tunnel. Our results reveal an increase in the lift to drag (L/D) ratio of the wing by $25 \%$ and a delay in the model's stall angle by $+6^{\circ}$ compared to a monoplane; implying a lower stalling speed for mini unmanned aerial vehicles (UAVs) and MAVs. These advancements if applied could revolutionize the capabilities of intelligent flying systems by enabling them to carry better sensors, computational units and other payloads as per the mission.
\end{abstract}

KEYWORDS: MAV, Box Wing, Stall Characteristics

\section{INTRODUCTION}

The progress of current technology has enabled the growth of small unmanned aerial vehicles or Micro Aerial Vehicles (MAV). These are being increasingly used for Agriculture, Search and Rescue, Military Reconnaissance and Surveillance operations due to their agility and ability to perform remote actions keeping human personnel away from harm. When it comes to fixed-wing designs, the capability to carry more payload, have a larger endurance and perform quick manoeuvres are desirable. Non-Conventional wing designs enable better drag reduction as compared to conventional wing designs of the same span (Kroo I., 2005). Also, they offer the possibility of reducing the aircraft weight, stall speed and increasing endurance. The minimum vortex drag for systems with the same geometric span and carrying to same total lift is shown in Figure 1 from Kroo I. (2005) for biplanes, box planes, a ring-wing, and winglets with varying ratios of height to span. The box plane achieves the lowest drag for a given span and height. In Figure 2 from Kroo I. (2005), the span efficiency (induced drag of planar wing / induced drag of the non-planar system of the same span and lift) for several nonplanar geometries is written alongside the configurations. 
Several non-aerodynamic features are of interest as well including effects on stability and control, characteristics of wake vortices, and structural implications of the nonplanar design but we will not be studying them here.

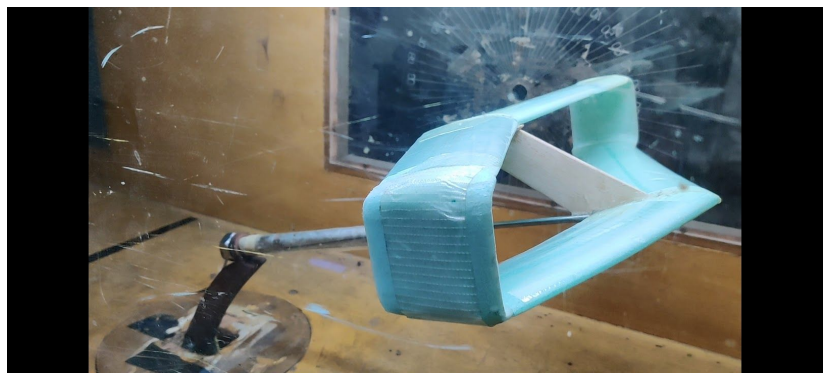

Fig. 1 : Box wing scaled model in the wind tunnel.

\subsection{Related Work}

L. Prandtl in 1942 suggested the "Best Wing System" where the lifting system with minimum induced drag is a proper box-like wing, in which the following conditions are satisfied: same lift distribution and same total lift on each of the horizontal wings and butterfly-shaped lift distribution on the vertical tip wings (Fig 4 from Frediani, A., 2005). When this occurs, the velocity induced by the free vortices is constant along the two horizontal wings and identically zero on the vertical side wings (Frediani, A., 2005). The relation between the induced drag of the box wing divided by the induced drag of a conventional wing and gap to span ratio $(\mathrm{h} / \mathrm{b})$ was further explored by Frediani and Montanari. The box wing design was adapted for transonic transport aircraft at Lockheed in the early 1970s by Miranda (1973) and Lange et al (1974). De Vivo, L et al (2019) have attempted to design, optimize, and prototype a box wing for a Low-Altitude Long-Endurance (LALE) UAV platform. The goal was to extend imaging campaigns and beyond-horizon communication in support of remote exploration, environmental assessment, disaster, post-disaster reconnaissance, and assistance using a Canard Prandtl Box Wing design. Jemitola P.O. et al (2012) performed a conceptual design of a 270 single class passenger capacity box wing aircraft with a design range of $4000 \mathrm{~nm}$ at Mach 0.8 , cruise altitude of $36,000 \mathrm{ft}$ and maximum takeoff distance of $2500 \mathrm{~m}$. Schiktanz, D. et al. (2011) have given a summary of geometric definitions of box wing configurations which are necessary to determine its aerodynamic characteristics and assess induced drag. A distinction was made between the aspect ratio of a single wing as compared to the whole wing configuration to justify the effects of the induced drag. The approach using the whole wing configuration was most suitable as it made possible to determine the span efficiency of the aircraft with the help of literature data. The vertical gap between the wingtips is crucial for span efficiency but the average $\mathrm{h} / \mathrm{b}$ ratio is only determined for standard wings with no dihedral.

Khan, F.A. \& Krammer, P. (2010) concluded that for a box wing stagger, sweep and taper ratio have no effect on the complete induced drag if adequate span loading is maintained. When dihedral is introduced in the system, induced drag increases and height to span ratio was the most important variable influencing the efficiency. A Ribeiro, F.C. et al. (2017) have studied the aerodynamics effects of a zero sweep, dihedral and twist Box Wing Aircraft's geometric parameters and compared aerodynamic efficiency between the Box Wing and a conventional mid-range size aircraft (Fig 2 from Ribeiro, F.C.). The results show for $h / b<0.2$, the gap to span ratio parameter and aspect ratio are important in the induced drag but higher values of $\mathrm{h} / \mathrm{b}$ offer less induced drag when taper ratio increases. For a small UAV carrying miniature computers and peripherals, electronic sensors, and optical sensing equipment, a non-planar box wing model can achieve some of the desired goals of a Small Reconnaissance Surveillance and Target Acquisition UAV (Landolfo, G. \& Altman, A. 2009).

There is indeed scope for box wing designs to be explored for MAV applications. It has potential in both Aerodynamic and Structural Domains. 


\section{AIM OF STUDY}

In our study, we aim to determine the enhancement of stall characteristics of Box Wing for MAVs, verify if the box wing design provides a better Lift to Drag (L/D) ratio as compared to conventional wings. Selection of Airfoil and designing the dimensions for the Wing. Further, CAD Model construction and optimisation and aerodynamic analysis by performing Wind Tunnel Tests and CFD. Stall observation is done using qualitative and quantitative methods.

\section{METHODOLOGY}

\subsection{Theoretical calculations}

Airfoils that exhibited good performance when flying at low Reynolds Numbers were explored. For the study, the research space was bound from 100,000 to 500,000. Clark Y airfoil had established capabilities in terms of Aerodynamic performance. It was an airfoil with high $\mathrm{C}_{\mathrm{L}} / \mathrm{C}_{\mathrm{D}}$ ratio having a max $\mathrm{C}_{\mathrm{L}} / \mathrm{C}_{\mathrm{D}}$ ratio of 95.0 (De Vivo, L. et al, 2019). The aerodynamic properties of Clark Y airfoil are listed in Table 1.

\subsection{Final Design}

Mean Aerodynamic Chord Calculations: The theory of the lifting line for a finite wing and MAC line was used to estimate the lift of the wing. Oswald's factor was assumed to be 0.8 (i.e. e $=0.8$ ).

Table 1 Clark Y Airfoil Info Table (Source: De Vivo, L. et al)

\begin{tabular}{|l|l|l|l|}
\hline Parameter & Value & Parameter & Value \\
\hline Thickness & $11.71 \%$ & Constant Parasitic Drag & 0.007 \\
\hline Max. Thick Pos & $28.28 \%$ & Maximum Section Lift Coefficient & 1.43 \\
\hline Max. Camber & $3.43 \%$ & Max Cl/Cd & 95.0 \\
\hline Max Camber Pos & $42.42 \%$ & Optimal AoA @ Max Cl/Cd & 3.6 deg \\
\hline Zero Lift Incidence & -3.68 & Cl @ Optimal AoA & 0.8 \\
\hline Zero Incidence Lift & 0.41 & Cd @ Optimal AoA & 0.008 \\
\hline Section Pitching moment Coefficient & -0.086 & - & \\
\hline
\end{tabular}

Table 2 Data of Both Aircraft

\begin{tabular}{|l|l|l|}
\hline Airfoil used: & Clark Y & \\
\hline Material used: & Styrofoam & \\
\hline Monowing: & Front wing & Rear wing \\
\hline Wing area: & $2.8 \mathrm{dm}^{2}$ & - \\
\hline MAC length: & $70.48 \mathrm{~mm}$ & - \\
\hline MAC distance: & $96.24 \mathrm{~mm}$ & - \\
\hline CG: & $31.41 \mathrm{~mm}$ from leading edge at the root & - \\
\hline Wing-span & $430 \mathrm{~mm}$ & - \\
\hline Taper ratio: & $3: 4$ & - \\
\hline Swept angle & 25 degrees & - \\
\hline
\end{tabular}




\begin{tabular}{|l|l|l|}
\hline Box Wing & Front wing & Rear wing \\
\hline Wing area: & $2.8 \mathrm{dm}^{2}$ & $2.8 \mathrm{dm}^{2}$ \\
\hline MAC length: & $70.48 \mathrm{~mm}$ & $70.48 \mathrm{~mm}$ \\
\hline MAC distance: & $96.24 \mathrm{~mm}$ & $96.24 \mathrm{~mm}$ \\
\hline CG: & $31.41 \mathrm{~mm}$ & $59.6 \mathrm{~mm}$ \\
\hline Wing-span & $430 \mathrm{~mm}$ & $430 \mathrm{~mm}$ \\
\hline Taper ratio: & $3: 4$ & $3: 4$ \\
\hline Swept angle & 25 degrees & 25 degrees \\
\hline
\end{tabular}

\subsection{CFD Simulation}

The vortex core analysis was done and the result is shown in the figure given below. Stalling Angle from the simulation: 16.04 degrees, $\mathrm{C}_{\mathrm{Lmax}}: 0.612$.

\subsection{Experiment on the real model}

To ensure the consistency between the theoretical findings, experimental tests were conducted in both qualitative and quantitative domains. The experimental models were fabricated indigenously using conventional aeromodelling techniques. High-density styrofoam was used as the base material and it was shaped with aluminium guide supports using a hot wire cutter. Finer adjustments in the airfoil profile were made using abrasive paper and ensuring the correct Clark Y profile. An online plotter was used to obtain the coordinates for airfoil which were stencilled on an aluminium sheet for cutting out the guide supports.

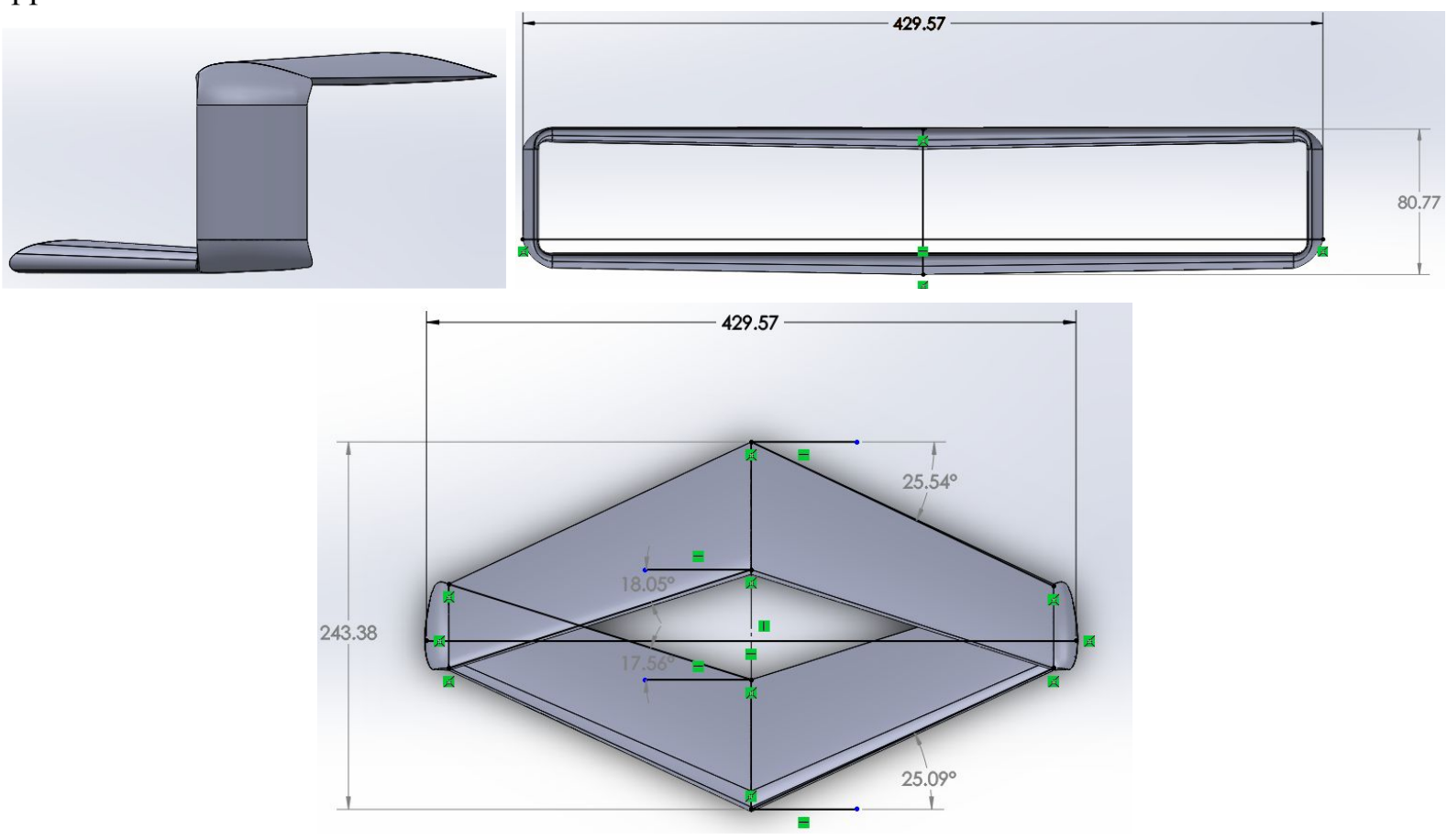

Fig. $1 \mathrm{CAD}$ of the Final Design of the box wing (all dimensions in $\mathrm{mm}$ ) 


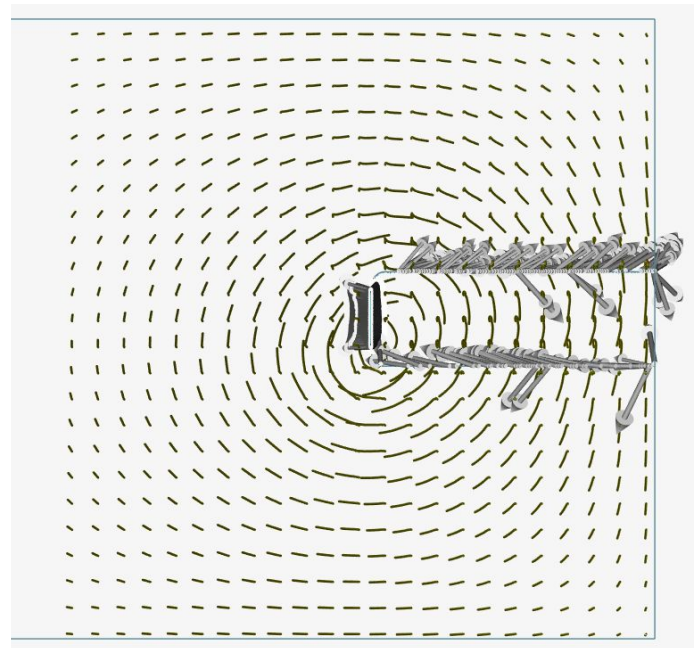

Fig 2 CFD analysis of vortices at the winglets/wing plates for a half box wing

The tests were conducted in the suction-type low-speed subsonic wind tunnel which has a test section size of $61 \mathrm{~cm} \times 61 \mathrm{~cm}$. The wind speed in the test section may vary from $18 \mathrm{~m} / \mathrm{sec}$ to $35 \mathrm{~m} / \mathrm{sec}$. Experiments related to the measurement of surface pressure distribution as well as overall forces and moments on a variety of models are conducted in the tunnel. The following sections describe the experimental work performed on the wind tunnel.

\subsubsection{Force measurement and comparison between monoplane and boxwing:}

Force measurement was performed using a 6 component strain gauge sting balance. To determine the flow velocity, the pressure difference between the settling chamber and the test section is measured. While comparing the aerodynamics parameters, i.e. $\mathbf{C}_{\mathbf{L}}, \mathbf{C}_{\mathbf{D}}$, $\mathbf{E}$, etc. it is ensured to have the same freestream velocity of $18 \mathrm{~m} / \mathrm{s}$.

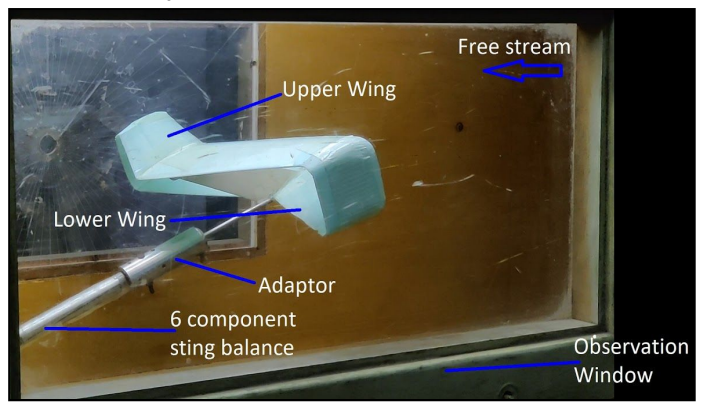

Fig 3. Experimental Setup in Wind Tunnel

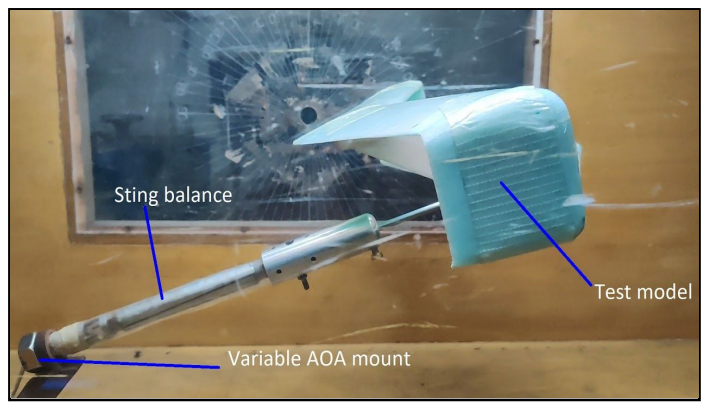

Fig 4. Model Mounted with Sting Balance

Model of the conventional wing was mounted on the variable angle of attack mount and the angle of attack was increased from 0 to $14 \mathrm{deg}$. The stall was observed at about $12 \mathrm{deg}$. Similarly, the box wing was mounted in the tunnel and angle of attack was increased from -5 to $15 \mathrm{deg}$. For the box wing, stalling was observed at $15 \mathrm{deg}$. 

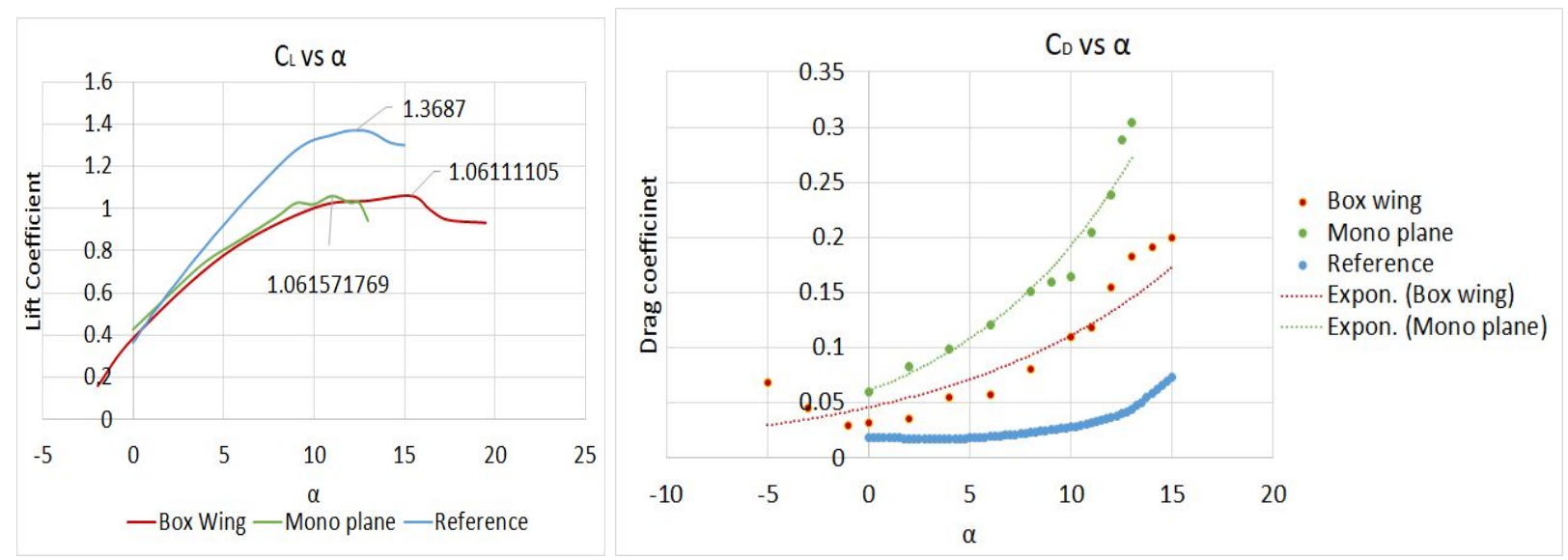

Fig. 5: Lift Coefficient vs. a on left and Drag Coefficient vs. a on right

\subsubsection{Tuft flow analysis}

To analyse the qualitative trends and local flow field features of the flow, polyester thread tufts were used. The wing was equipped with tufts of polyester fibre by placing them uniformly in three equispaced rows on the upper surfaces of both the upper and lower wing. Two rows of tufts were placed on the outer surface of each winglet. On mounting the augmented wing in the tunnel the angle was increased step by step and timely observed phenomena of flow separation and recirculation very clearly. Once recirculation was observed on both the wings, the angle was noted around $15 \mathrm{deg}$.
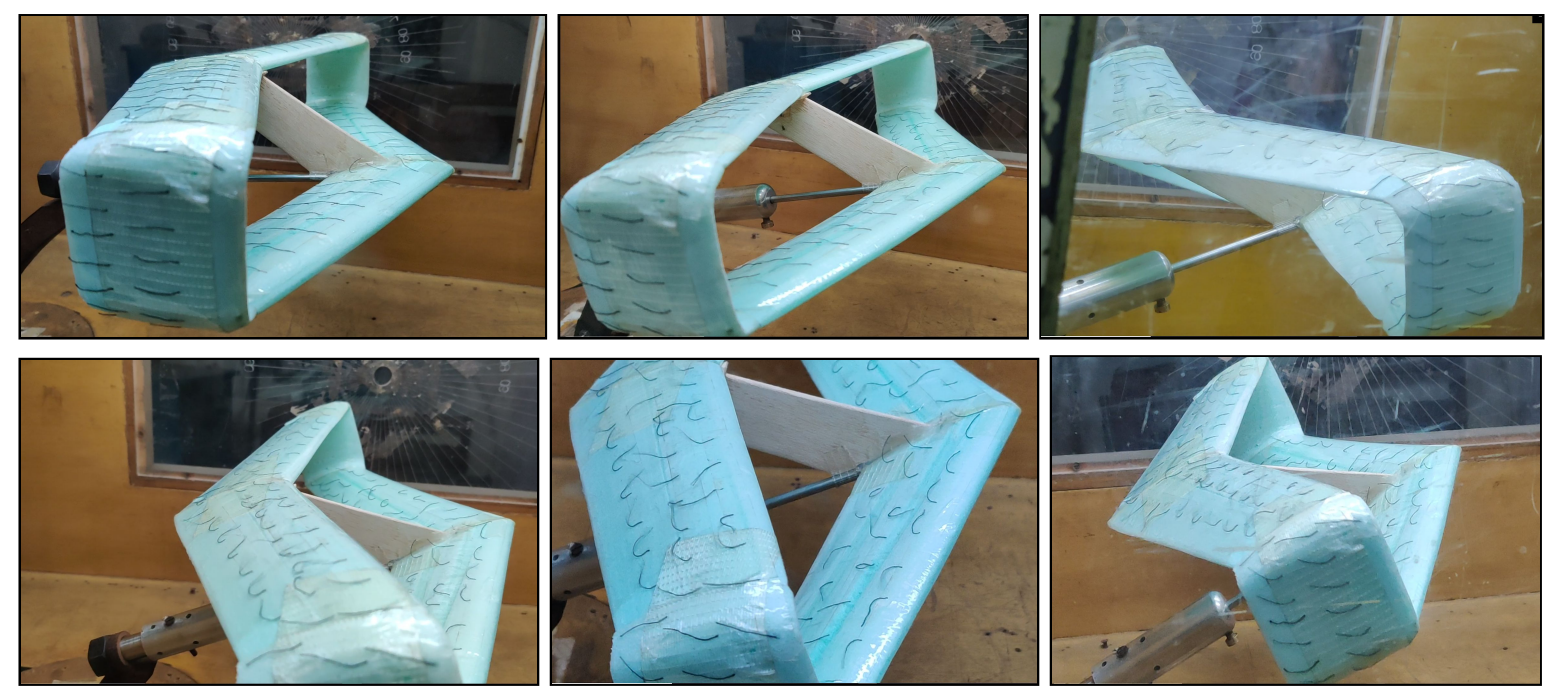

Fig. 6 Tuft Flow Analysis - Clockwise from top left: angles- 0,5,10, 10, 15, 20 degrees

\subsubsection{Smoke flow visualisation}

The previous test was extremely essential to analyse the local flow field. However, the flow characteristics in the wake of the box wing model cannot be determined. Thus, to study it experimentally smoke flow visualisation was used. Similar to the previous test, the model was mounted inside the tunnel 
at AOA 0 deg. The settling chamber of the tunnel was filled with smoke for a considerable period of time. The tunnel was started at a free stream velocity of $18 \mathrm{~m} / \mathrm{s}$ and a laser sheet was projected parallel to the cross-section of the tunnel to closely observe the test section for the flow properties. After considering still visuals from numerous tests, concluded this test could not reflect the actual scenario in the best manner and we could not derive a meaningful conclusion from the smoke flow analyses. This can be majorly due to the dimensions of the model, discontinuities of smoke in streaklines, freestream velocity of $18 \mathrm{~m} / \mathrm{s}$.

\section{CONCLUSION}

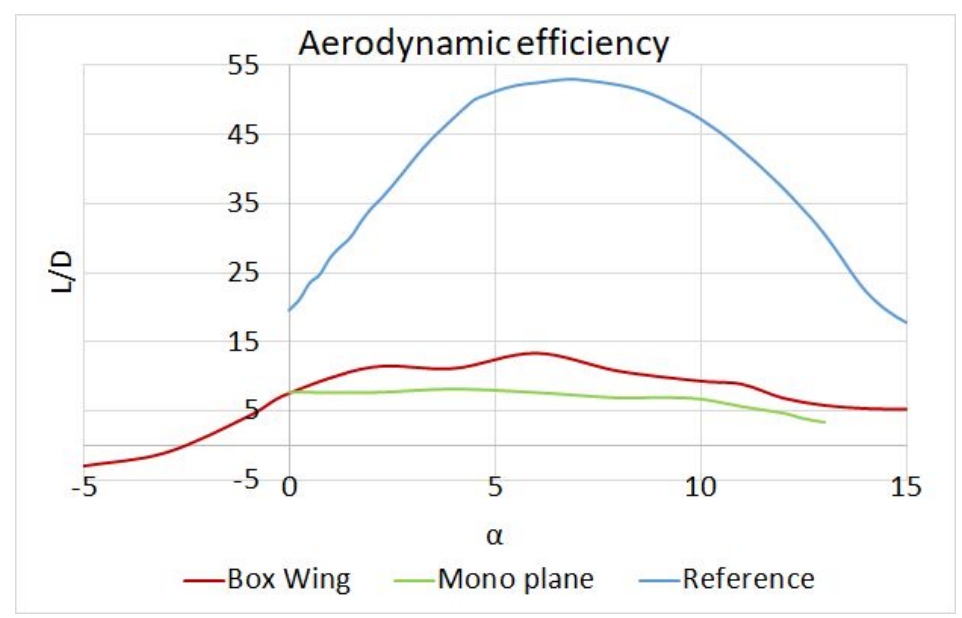

Fig7: Aerodynamic efficiency vs. a

In this paper, a box wing model was designed and developed for applications in MAVs. Theoretical calculations were performed and the wing parameters were set in a CAD model. CFD Simulations were done to understand the range of values for the lift and drag forces and to estimate the stalling characteristics. Three kinds of experiments were performed in the wind tunnel- Force measurement using Sting balance, Tuft Flow Analysis and Smoke flow Visualisation. Considering the CFD results, force measurement and tuft flow results, we conclude that the box wings showcase a considerable delay in the stalling. Observations have also shown that in the case of the box wing, the lift to drag ratio is higher indicating a better aerodynamic efficiency when compared to the monoplane wing.

The optimisation of the design is of primary concern to enable it to achieve flight. The correct $\mathrm{h} / \mathrm{b}$ ratio can ensure minimum induced drag and maximise the efficiency of the aircraft. Further studies would be done to enable good gust performance and static and dynamic stability of the aircraft.

\section{REFERENCES}

De Vivo, L., Tran, D., Kuester, F. (2019). Towards Design of a 3D Printable Prandtl Box-Wing Unmanned Aerial Vehicle. In 2019 IEEE Aerospace Conference.

Frediani, A. (2005). THE PRANDTL WING. VKI, lecture series on Innovative Configurations and Advanced Concepts for Future Civil Transport Aircraft.

Frediani, A., \& Montanari, G. (2009). Best Wing System: An Exact Solution of the Prandtl's Problem. Variational Analysis and Aerospace Engineering. (Vol. 33.pp. 183-21).

Jemitola, P.O., Fielding, J.P. (2012). BOX WING AIRCRAFT CONCEPTUAL DESIGN. 28th International Congress of the Aeronautical Sciences. 
Khalid, A., Golson, B. (2014). Aerodynamic Analysis of Box Wing Configuration for Unmanned Aircraft System (UAS) - Student Design Project. 2014 ASEE Southeast Section Conference.

Khan, F.A., Krammer, P. (2010). Preliminary Aerodynamic Investigation of Box-Wing. Configurations using Low Fidelity Codes.

Kroo, I. (2005). Nonplanar Wing Concepts For Increased Aircraft Efficiency. VKI lecture series on Innovative Configurations and Advanced Concepts for Future Civil Aircraft.

Landolfo, G. \& Altman, A. (2009). Aerodynamic and Structural Design of a Small Nonplanar Wing UAV. 47th AIAA Aerospace Sciences Meeting Including The New Horizons Forum and Aerospace Exposition.

Miranda, L. (1973). Boxplane wing and aircraft. US Patent US3834654A. Lockheed Corp.

Prandtl, L. (1924). Induced drag of multiplanes. NACA TN 182.

Ribeiro, F.C., de Paula, A.A., Scholz, D., \& da Silva, R.G.A. (2017). Wing geometric parameter studies of a box wing aircraft configuration for subsonic flight. 7th European Conference For Aeronautics And Space Sciences (EUCASS).

Schiktanz, D., Scholz, D. (2011). BOX WING FUNDAMENTALS- An Aircraft Design Perspective. German Aerospace Conference 2011, At Bremen, Germany (Volume: pp 601-615). 